\title{
Pacientes com depressão maior têm menor sensibilidade a contraste visual que indivíduos saudáveis
}

\author{
Renata Maria Toscano Barreto Lyra Nogueira \\ Universidade Federal de Alagoas \\ Natanael Antonio dos Santos \\ Universidade Federal da Paraíba
}

\begin{abstract}
Resumo
O objetivo foi medir a sensibilidade ao contraste, $\mathrm{SC}$, visual de grades senoidais circulares concêntricas com frequências espaciais de 0,$25 ; 4$ e $8 \mathrm{cpg}$, ciclos por grau de ângulo visual, em adultos saudáveis e com depressão maior. Foram estimadas a SC de 20 participantes, 10 saudáveis e 10 participantes com depressão maior, utilizando o método psicofísico da escolha forçada e luminância média de $0,7 \mathrm{~cd} / \mathrm{m}^{2}$. Todos os participantes apresentavam acuidade visual normal ou corrigida e estavam livres de doenças identificáveis. Os resultados mostraram que a SC visual máxima ocorreu na faixa de 0,25 cpg para os dois grupos. Os resultados demonstraram ainda que a $\mathrm{SC}$ visual dos participantes com depressão maior foi mais baixa do que a dos participantes saudáveis $(p<0,05)$, pois os participantes com transtorno precisaram de mais contraste para detectar as frequências espaciais testadas. Estes resultados sugerem alterações na percepção visual relacionadas à depressão maior.
\end{abstract}

Palavras-chave: percepção visual; sensibilidade ao contrate; frequências espaciais; método psicofísico.

\begin{abstract}
Patients with major depression have visual contrast sensitivity less than healthy subjects. The aim of this study was to measure visual contrast sensitivity, CS, for concentric circular sine-wave gratings with spatial frequencies of $0.25,4$ and $8 \mathrm{cpd}$, cycles per degree of visual angle, in adults without and with major depression disorder. We measured the visual CS for 20 participants, ten without and ten with major depression disorder medicated, using a temporal two-alternative forced-choice psychophysical method with mean luminance of $0.7 \mathrm{~cd} / \mathrm{m}^{2}$. All volunteers were free from identifiable ocular disease and had normal acuity. The results showed maximum contrast sensitivity to spatial frequency occurred in the range of $0.25 \mathrm{cpd}$ for both groups. The results also showed that the visual CS for major depression was lower than those of adults without depression $(p<0.05)$. These results showed alterations in the visual perception related the major depression.

Keywords: vision perception; contrast sensitivity; spatial frequency; psychophysical method.
\end{abstract}

$\mathrm{A}$ depressão maior pode ser caracterizada como um transtorno de humor crônico, recorrente e debilitante que traz prejuízos no funcionamento e na qualidade de vida dos pacientes acometidos (Baptista, 2004; Calil \& Guerra, 2004; Moreno \& Soares, 2003; Solomon, 2002). A depressão tem sido associada a prejuízos cognitivos e neurofisiológicos diversos (Dalgalarrondo, 2008; Licinio \& Wong, 2007). A incidência anual da depressão torna-a um dos problemas de saúde pública mais importante, com repercussões socioeconômicas severas, com altos custos em assistência médica e redução da qualidade de vida (Solomon, 2002). De acordo com o relatório da Organização Mundial de Saúde (World Health Report [WHO], 2001), a depressão maior é uma das principais causas de incapacitação em todo o mundo.

Pesquisar o sistema visual de pacientes com depressão maior com métodos psicofísicos e estímulos diferentes processados por áreas visuais superiores pode complementar a investigação clínica e contribuir para a caracterização de mecanismos cognitivos, afetivos, e neurofisiológicos básicos relacionados a esta patologia. Pode revelar, inclusive, novos aspectos teóricos, comportamentais e funcionais da depressão em geral e apontar meios alternativos para o diagnóstico.

A sensibilidade ao contraste, $\mathrm{SC}$ visual, é uma ferramenta muito utilizada para avaliar e diagnosticar alterações decorrentes de anormalidades na retina (Owsley et al., 2000), vias e áreas visuais superiores (Wesner \& Tan, 2006) e até mesmo transtornos que não estão associados diretamente às funções visuais, como por exemplo, esquizofrenia (Butler, Silverstein, \& Dakine, 2008); diabete (Gualtirei et al., 2011); epilepsia (Bezerra, Alencar, Mousinho, \& Santos, 2011); desnutrição (Santos \& Alencar, 2010); doenças de Alzheimer e de Parkinson (Elliott \& Situ, 1998; Polat, Sagi, \& Norcia, 1997; Vleugels, Van Nunen, 
Lafosse, Ketelaer, \& Vandenbussche, 1998; Silva et al., 2005), dentre outras.

Pesquisas relacionando a SC visual e transtornos depressivos ainda são reduzidas e os poucos trabalhos encontrados na literatura foram realizados com estímulos, populações e propósitos distintos (Bubl, Tebartz, Gondan, Ebert, \& Greenlee, 2009; Szabó et al., 2004; Wesner \& Tan, 2006). Por exemplo, Szabó et al. (2004) mediram a SC visual de voluntários normais e com desordem afetiva sazonal, utilizando grade senoidal linear estática e dinâmica com frequências de 0,5 a 14,4 cpg, ciclos por grau de ângulo visual. O objetivo da pesquisa era investigar os efeitos da terapia à base de luz no tratamento da depressão sazonal. Os resultados mostraram que a terapia a base de luz melhora de forma significante a SC visual dos pacientes nas frequências espaciais estáticas abaixo de $5,7 \mathrm{cpg}$.

Cavalcanti e Santos (2007), em um estudo piloto, mediram o limiar de contraste de voluntários adultos sem e com depressão maior, utilizando estímulos visuais radiais estáticos com frequência espacial de 0,2 a 4 cpg. Os resultados demonstraram que os pacientes com depressão maior medicados apresentaram prejuízos, ou seja, menor SC para todas as frequências quando comparados aos voluntários sem transtornos neuropsiquiátricos.

Por fim, Wesner e Tan (2006) mediram o limiar de contraste de pacientes com depressão sazonal, com depressão não sazonal e sem depressão, utilizando estímulos visuais estáticos e dinâmicos, modulados pela função de Gabor, com frequências espaciais de 0,3 a 12 cpg, em condições fotópicas. Os resultados mostraram que os participantes com depressão apresentam maior SC visual do que o grupo sem depressão nas frequências espaciais estáticas de 6 e $12 \mathrm{cpg}$. Os autores discutem que a depressão pode acentuar a SC visual para as frequências que são processadas pela via visual parvocelular.

O presente estudo teve como objetivo medir a SC visual acromática de voluntários adultos sem depressão e com depressão maior, utilizando grades senoidais circularmente concêntricas e estáticas com frequências espaciais de 0,25, 4 e $8 \mathrm{cpg}$ (Figura 1) com luminância média de $0,7 \mathrm{~cd} / \mathrm{m}^{2}$. A hipótese de pesquisa foi investigar se a depressão maior altera a resposta, $\mathrm{SC}$, do sistema visual ou os mecanismos sensoriais básicos envolvidos no processamento de padrões visuais formados por grades senoidais concêntricas estáticas com frequências de $0,25,4$ e 8 cpg. O foco principal foi à comparação entre grupos para as mesmas frequências, já que as frequências espaciais são diferentes e tendem a gerar resultados diferentes e interação entre grupo e frequência. As medidas foram realizadas com o método psicofísico da escolha forçada temporal entre duas alternativas, um paradigma bem estabelecido que minimiza os efeitos do critério de escolha de resposta dos participantes, um dos problemas dos métodos psicofísicos clássicos, por exemplo, métodos dos limites, do ajuste e dos estímulos constantes.

Esse estudo foi motivado pelo pressuposto que certos transtornos neuropsiquiátricos como a depressão e a esquizofrenia alteram o sistema nervoso central (Kéri, Antal, Szekeres, Benedek, \& Janka, 2000; Slaghuis, 1998; Wesner \& Tan, 2006) e podem atuar em uma série de disfunções sensoriais, cognitivas e emocionais promovendo dentre outros aspectos distorções das vias visuais (Butler et al., 2008; Kéri et al., 2000; Wesner \& Tan, 2006). A SC visual é uma ferramenta objetiva, não invasiva, muito utilizada na clínica que pode contribuir com um maior entendimento dos mecanismos sensoriais associados aos sintomas da depressão. A depressão maior é um transtorno recorrente de difícil diagnóstico e a $\mathrm{SC}$ visual pode contribuir para o diagnóstico objetivo da doença. Além disso, os estudos encontrados e descritos foram realizados com grade senoidal em coordenadas cartesianas, exceto o estudo Piloto de Cavalcanti e Santos (2007) que utilizou grade não cartesiana, mas com amostra menor e grades senoidais concêntricas com frequências diferentes das utilizadas no presente estudo. O processamento visual de grades senoidais circularmente concêntricas tem sido associado a regiões corticais extra-estriados, por exemplo, a área visual V4 (Gallant, Braun, \& Van Essen, 1993). Esses autores registraram as respostas de 181 células em V4 e verificaram que a maior parte das células responde melhor para estímulos circularmente concêntricos em comparação a grades senoidais lineares e estímulos hiperbólicos.

\section{Método}

\section{Participantes}

Participaram do estudo 20 voluntários adultos de ambos os sexos, com idade entre 20 e 30 anos, dez saudáveis $(M=$ $22,8 ; D P=1,2)$ e dez diagnosticados com depressão maior $(M=24,1 ; D P=3,1)$, de acordo com critérios do Manual Estatístico e Diagnóstico de Transtornos Mentais, DSM IV (American Psychiatric Association, [APA] 2005). Os pacientes com depressão maior foram diagnosticados por psiquiatras do Centro de Atenção Psicossocial, CAPS, e encaminhados ao laboratório, onde foram realizados os testes visuais. Todos os participantes preencheram o Inventário de Depressão de Beck, BDI, e uma entrevista semiestruturada. A entrevista foi utilizada para eliminar fumantes ou participantes que apresentavam alguma patologia identificável. Já o BDI foi usado para ajudar na classificação dos participantes saudáveis e com depressão. As amostras do estudo foram compostas por pessoas jovens para evitar a presença de transtornos neurológicos.

Dos dez pacientes com depressão maior, sete eram medicados, cinco usavam antidepressivos inibidores seletivos da recaptação de serotonina, um usava antipsicótico (Risperidona), um usava concomitantemente antidepressivo (Venlafaxina), ansiolítico (Clonazepan) e hipnótico (Zolpidem). Dos outros três, um usava floral de Bach e dois não usavam nenhum fármaco. Todos os voluntários apresentavam acuidade visual normal ou corrigida, testados com as cartelas de optotipos direcionais "E” de Rasquin, e não tinham história de patologia ocular ou neurológica.

A participação na pesquisa ocorreu mediante assinatura de Termo de Consentimento Livre e Esclarecido conforme a Resolução nº 196/96 do Conselho Nacional de Saúde, Ministério da Saúde, que trata das diretrizes e normas de pesquisas envolvendo seres humanos. A pesquisa foi aprovada pelo Comitê de Ética do Centro de Ciências da Saúde. 


\section{Equipamento e estímulos}

Os estímulos visuais foram gerados em um monitor de vídeo CRT (Cathodic Ray Tube) CLINTON MEDICAL monocromático de 21 polegadas, digital e com resolução da tela 1024 x 768 pixels, frequência de atualização da imagem de $70 \mathrm{~Hz}$, controlado por um microcomputador. Uma cadeira foi fixada a $150 \mathrm{~cm}$ da tela do monitor de vídeo, com apoio de queixo e testa para controlar a distância. Um programa LightScan com um fotômetro OptiCAL (Cambridge Research Systems, Rochester, Kent) foi utilizado para medir a luminância média da tela do monitor $\left(0,7 \mathrm{~cd} / \mathrm{m}^{2}\right)$. A luminância mínima foi de $0,5 \mathrm{~cd} / \mathrm{m}^{2}$ e a máxima de $0,9 \mathrm{~cd} / \mathrm{m}^{2}$. O ambiente do laboratório era cinza para melhor controle da luminância.

Foram utilizados estímulos visuais formado por grade senoidal circularmente concêntrica e estática, com frequências espaciais de 0,$25 ; 4$ e 8 ciclos por grau de ângulo visual, cpg
(Figura 1). Deu-se preferência a frequências espaciais entre 0,25 e $8 \mathrm{cpg}$ devido às condições baixas de luminância utilizadas no experimento que podem ser consideradas como mesópica. Entende-se por luminância mesópica, a luminância que varia aproximadamente entre 0,003 a $3 \mathrm{~cd} / \mathrm{m}^{2}$, que está situada entre a condição escotópica e a fotópica e que provavelmente ativa tanto os cones como os bastonetes (Ketomäki, Eloholma, Orreveteläinen, \& Halonen, 2003).

Estes eram circulares com um diâmetro de 7 graus de ângulo visual a $150 \mathrm{~cm}$ de distância. $\mathrm{O}$ estímulo neutro era cinza, homogêneo e apenas continha a luminância média. Todos os estímulos eram acromáticos e foram apresentados em tempo real no centro do monitor. Um programa desenvolvido pelo próprio grupo, escrito em linguagem $\mathrm{C}++$, foi utilizado para rodar os experimentos. Isto é, gerar os estímulos, controlar a apresentação dos estímulos e registrar os limiares de contraste.
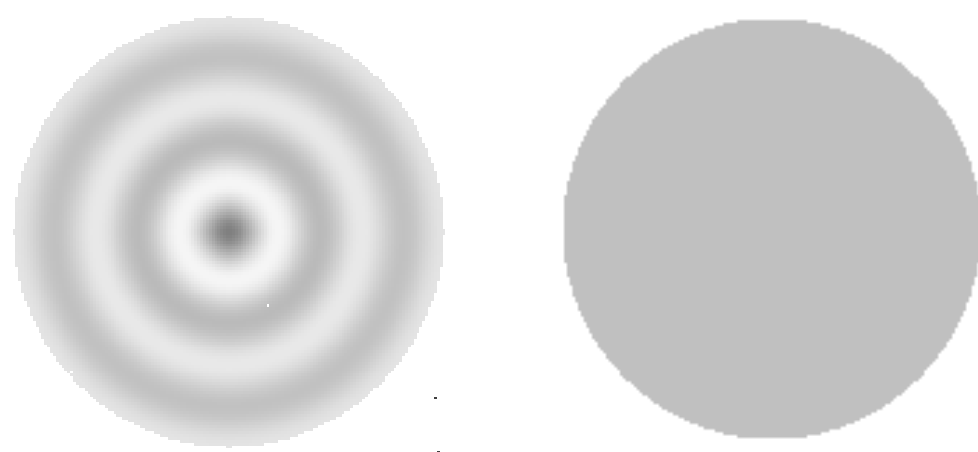

Figura 1. Exemplos de um par de estímulos, a esquerda uma grade senoidal concêntrica com frequência espacial de $1 \mathrm{cpg}$ (ciclos por grau de ângulo visual) e a direita um estímulo neutro. Estímulos originalmente calibrados para serem vistos a $150 \mathrm{~cm}$ de distância antes de fotografados.

\section{Procedimento}

As medidas de SC visual foram realizadas com o método psicofísico da escolha forçada temporal entre duas alternativas (Wetherill \& Levitt, 1965). Este método se baseia no cálculo da probabilidade de acertos consecutivos por parte do voluntário, ou seja, em cerca de 100 apresentações de escolhas entre os dois estímulos (estímulo de teste e estímulo neutro), a frequência espacial (estímulo de teste) deve ser percebida $79 \%$ das vezes pelo voluntário.

O procedimento para medir o limiar para cada frequência consistiu na apresentação sucessiva simples do par de estímulos, no qual um deles era o estímulo teste, que deveria ser identificado pelo participante. $\mathrm{O}$ critério adotado para variar o contraste de cada frequência espacial testada foi o de três acertos consecutivos para decrescer uma unidade e um erro para acrescer da mesma unidade $(0,08 \%)$. O contraste foi definido pela fórmula de Michelson.

Durante cada sessão experimental foi apresentada uma sequência de pares de estímulos iniciada com um sinal sonoro acompanhado imediatamente pela apresentação do primeiro estímulo por $2 \mathrm{~s}$, seguido de um intervalo entre estímulos de 1 $\mathrm{s}$ e pela apresentação do segundo estímulo por $2 \mathrm{~s}$ e da resposta do participante. A ordem de apresentação dos estímulos era aleatória. Os participantes foram instruídos a pressionar o botão do lado esquerdo do mouse quando a grade senoidal concêntrica ou frequência espacial era apresentada primeiro e o botão do lado direito quando era apresentada em segundo lugar, isto é, após o estímulo neutro. Se a resposta do voluntário fosse correta, era seguida por outro sinal sonoro e um intervalo de $3 \mathrm{~s}$ para a sequência se repetir. $O$ sinal sonoro que indicava o início da apresentação do par de estímulos e o que indicava a escolha correta eram diferentes. A duração da sessão experimental variou entre 5 e $10 \mathrm{~min}$, dependendo dos erros e acertos do participante até proporcionarem um total de seis reversões ou seis valores de contraste conforme requerido para o final automático da mesma.

Cada uma das frequências da curva de SC visual foi estimada pelo menos duas vezes ou duas sessões experimentais, em dias diferentes, por cada um dos participantes. Em média, doze curvas foram medidas para cada grupo de voluntários, como cada curva era formada por três frequências espaciais, totalizou-se 60 sessões experimentais para cada grupo. Todas as estimativas foram medidas à distância de $150 \mathrm{~cm}$, com visão binocular e pupila natural.

Todos os participantes receberam a seguinte instrução: "será apresentada uma sequência de pares de figuras ou desenhos, uma com círculos claro-escuros e outra totalmente cinza. Você 
deverá escolher sempre a figura ou desenho que contém os círculos claro-escuros, pressionando o botão do lado esquerdo, botão número 1 do mouse quando o desenho com círculos for apresentado primeiro, e o botão do lado direito, botão número 2 , quando for apresentado em segundo lugar, após o desenho cinza".

É importante mencionar que cada sessão foi iniciada com o estímulo teste com contraste em nível supralimiar e que os experimentos só começaram quando o experimentador certificou-se que os participantes entenderam e responderam conforme as instruções. Neste sentido, a instrução foi repetida, em uma sessão de treino e ambientação com as condições experimentais, até os participantes compreenderem as instruções e discernirem entre o estímulo com círculos claro-escuros e o estímulo homogêneo em tom de cinza.

\section{Resultados}

Após cada sessão, o programa produziu uma folha de resultados com a situação experimental e os seis valores de limiar de contraste conseguidos pelas reversões. Os valores de limiar de contraste obtidos para cada frequência foram agrupados em planilhas por condição, com depressão e sem depressão, e foram transformados em SC (1/limiar de contraste). Em outras palavras, quanto menor o limiar de contraste maior a sensibilidade do sistema visual humano e vice-versa. Assim, os menores valores de limiares correspondem aos maiores valores de SC.

A Figura 2 mostra as curvas de $\mathrm{SC}$ visual obtidas através da média geral dos valores de SC de cada grupo: voluntários com depressão, C/D, e sem depressão, S/D. As barras verticais indicam os erros padrões das médias. A sensibilidade ao contraste visual média é apresentada em função das frequências espaciais.

Os resultados mostram que a $\mathrm{SC}$ visual máxima ocorreu na frequência espacial de 0,25 cpg para ambos os grupos (Figura 2). Entretanto, a SC visual do grupo com depressão, foi da ordem de $1,1,2,0$ e 1,8 vezes menos sensível para as frequências de 0,25 ; 4 e $8 \mathrm{cpg}$, respectivamente. A análise de variância (Repeated Measures ANOVA) revelou um efeito principal significante entre grupos $(F(1,358)=49,38 ; p<0,05)$, um efeito principal significante entre frequências $(F(2,358)=120,13 ; p<0,05)$, e uma interação significante de grupos versus frequências $(F(2$,

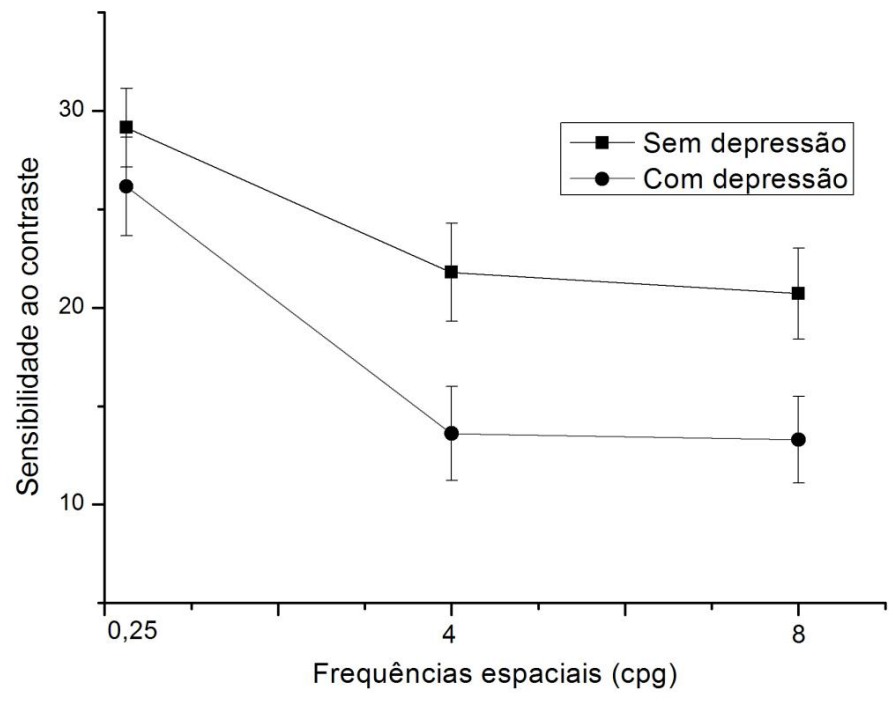

Figura 2. Curvas de sensibilidade ao contraste de participantes sem depressão e com depressão para grade senoidal concêntricas nas frequências espaciais de 0,$25 ; 4$ e 8 cpg (ciclos por grau de ângulo visual). As barras verticais indicam os erros padrões das médias.

$358)=36,32 ; p<0,05)$. As análises com o teste post-hoc Newman Kels mostraram diferenças significantes entre os dois grupos para as frequências de 4 e $8 \mathrm{cpg}(p<0,05)$. Estes resultados indicam que a depressão maior altera a SC visual, pois os participantes com depressão apresentaram menor sensibilidade (precisaram de mais contraste) para detectar grades senoidais concêntricas com frequências espaciais de 4 e $8 \mathrm{cpg}$, quando comparados aos participantes sem depressão.

\section{Discussão}

Este estudo mediu a SC visual acromática de participantes sem transtornos neuropsiquiátricos e pacientes com depressão maior, utilizando o método psicofísico da escada com escolha forçada temporal entre duas alternativas e níveis baixos de luminância.

Os resultados mostraram que a $\mathrm{SC}$ visual do grupo com depressão e do grupo sem depressão apresentam formas gerais semelhantes, isto é, máxima sensibilidade na faixa de frequência espacial de 0,25 cpg (Figura 2), com redução da SC nas faixas de frequências espaciais mais altas, 4 e $8 \mathrm{cpg}$. Entretanto, os portadores de depressão apresentaram prejuízos na SC visual, embora as alterações produzidas pela depressão sejam assimétricas; pois os mecanismos ou canais que processam as frequências de 0,$25 ; 4$ e 8 cpg em níveis baixos de luminância foram afetados de maneira diferente. O declínio observado 
entre os dois grupos na $\mathrm{SC}$ foi mais acentuado nas vias que provavelmente processam faixas de frequências mais altas (p.ex., 4 e $8 \mathrm{cpg}$ ), onde o grupo com depressão precisou de 2,0 e 1,8 vezes mais contraste para perceber os estímulos. Este resultado sugere um comprometimento maior da via parvocelular (Burbeck \& Kelly, 1981).

Não se sabe ao certo se a depressão altera as vias visuais magno e parvocelular de maneira distinta. Por exemplo, Szabó et al. (2004) relatam alterações na SC em frequências baixas como 0,5 cpg. Já Wesner e Tan (2006) discutem alterações na SC em frequências mais altas como $12 \mathrm{cpg}$. Wesner e Tan discutem que a depressão pode acentuar a SC para as frequências que são processadas pela via visual parvocelular. Esses estudos, incluindo a presente pesquisa, utilizaram estímulos e luminância diferentes, não sendo possível uma comparação direta entre os resultados. Logo, pesquisas futuras utilizando o mesmo método e estímulos em condições de luminâncias diferentes precisam ser realizadas para estudar de forma mais sistemática as alterações comportamentais nas vias visuais parvo e magnocelular relacionadas à depressão, já que a via visual parvocelular é especializada no processamento de frequências espaciais médias e altas ou detalhes finos dos objetos e opera em níveis altos ou fotópicos de luminância, e a via visual magnocelular é especializada no processamento de frequências espaciais baixas e opera em níveis baixos ou escotópicos de luminância (Banks \& Bennett, 1988; Brown, Dobson, \& Maier, 1987; Ellemberg, Lewis, Maurer, \& Brent, 2000; Hickey, 1977; Kiorpes \& Movshon, 1998; Van Sluyters, Atkinson, Held, Peter Hoffman, \& Shatz, 1990; Wilson, 1988; Youdelis \& Hendrickson, 1986).

Mudanças na SC visual relacionadas à depressão maior eram esperadas, considerando que alguns estudos relatam que os transtornos neuropsiquiátricos afetam a cognição e o funcionamento do sistema nervoso central (Ballone, Ortolani, \& Pereira, 2002; Bubl et al., 2009; Calil \& Guerra, 2004; Duma, Heninger, \& Nestler, 1997; Kaplan, Sadock, \& Sadock, 2007; Louzã-Neto, Motta, Wang, \& Elkis, 1995; O’Donnell et al., 2002; Rodrigues, 2000; Slaguis, 1998). Entretanto, os resultados obtidos no presente estudos ainda são iniciais, pois não encontramos nenhum estudo na literatura relacionando a $\mathrm{SC}$ visual ao transtorno depressivo, utilizando grades senoidais concêntricas estáticas em condições de baixa de luminância.

Além disso, é importante ressaltar que não se pode afirmar que o prejuízo encontrado na percepção ou SC visual seja fruto apenas de alterações produzidas pela depressão maior no sistema nervoso central. É provável que outros fatores estejam relacionados, como por exemplo, a influência dos antidepressivos no processamento visual de contraste, pois os antidepressivos atuam modulando o funcionamento do sistema nervoso central. Inclusive, existem indícios nos estudos que tentaram relacionar transtornos psicóticos e alterações na percepção visual, que o tipo de medicamento - antipsicótico, típicos ou atípicos - utilizado altera de forma distinta a percepção de contraste (Chen et al., 2003).

É importante ressaltar que o presente trabalho não teve o objetivo de controlar os efeitos dos antidepressivos nas medidas de SC visual, o que pode ser uma limitação do mesmo. Isso ocorreu porque os pacientes utilizavam medicamentos distintos, e não foi possível formar grupos baseados na medicação. Entretanto é preciso que a relação entre antidepressivos e alterações na SC visual seja investigada em estudos futuros, pois os resultados podem trazer avanços no diagnóstico e tratamento de um transtorno que possui extrema importância no contexto da atenção à saúde, por sua crescente prevalência na população geral, além de ter uma heterogeneidade na expressão clínica. É dentro desta perspectiva que novos estudos estão sendo conduzidos em nosso laboratório para avaliar o efeito da depressão e da medicação no processamento visual de estímulos diferentes em diferentes condições de luminância.

\section{Referências}

American Psychiatric Association [APA] (2005). DSM IV-TR: Diagnostic and Statiscal Manual of Mental Disorder. Washington: American Psychiatric.

Ballone, G. J., Ortolani, I. V., \& Pereira N. E. (2002). Da emoção à lesão. São Paulo: Manole.

Banks, M. S., \& Bennett, P. J. (1988). Optical and photoreceptor immaturities limit the spatial and chromatic vision of human neonates. Journal of the Optical Society of America A, 5, 2059-2079.

Baptista, N. M. (2004). Suicídio e depressão - Atualizações. Rio de Janeiro: Guanabara.

Bezerra, P. C., Alencar, C. C. G., Mousinho, S. R., \& Santos, N. A. (2011) Visual contrast sensitivity in tonic-clonic epileptic patients. Psychology \& Neuroscience, 4, 31-36. doi: 10.3922/j.psns.2011.?.00?

Brown, A. M., Dobson V., \& Maier, J. (1987). Visual acuity of human infants at scotopic, mesopic and photopic luminances. Vision Research, 27, 1845-1858.

Bubl, E., Tebartz, V. E. L., Gondan, M., Ebert, D., \& Greenlee, M. W. (2009). Vision in depressive disorder. World Journal of Biological Psychiatry, 10, 377-384. doi: 10.1080/15622970701513756

Burbeck, C. A., \& Kelly, D. H. (1981). Contrast gain measurements and the transient/sustained. Journal of the Optical Society of America A, 71, 13351342. doi: dx.doi.org/10.1364/JOSA.71.001335

Butler, P. D., Silverstein, S. M., \& Dakine, S. C. (2008). Visual perception and its impairment in schizophrenia. Biological Psychiatry, 64, 40-47. doi: 10.1016/j.biopsych.2008-03.023

Calil, H. M., \& Guerra, G. B. A. (2004). Depressão: uma doença mental? Ciência Hoje, 34, 28-33.

Cavalcanti, A. M., \& Santos, N. A. (2007). O efeito da depressão na função de sensibilidade ao contraste em humanos: achados preliminares. Revista Brasileira de Terapias Cognitivas, 1, 50-59.

Chen, Y., Levy, D. L., Sheremata, S., Nakayama, K., Matthysse, S., \& Holzman, P. S. (2003). Effects of typical, atypical and no antipsychotic drugs on visual contrast detection in schizophrenia. American Journal Psychiatry, 160, 1795-1801. doi: 10.1176/appi.ajp. 160.10.1795

Dalgalarrondo, P. (2008). Psicopatologia e semiologia dos transtornos mentais. São Paulo: Artes Médicas.

Duman, R. S., Heninger, G. R., \& Nestler, E. J. (1997). A molecular and cellular theory of depression. Archives of General Psychiatry, 54, 597-606. doi: 10.1001/archpsyc.1997.01830190015002

Ellemberg, D., Lewis, T. L., Maurer, D., \& Brent, H. P. (2000). Influence of monocular deprivation during infancy on the later development of spatial and temporal vision. Vision Research, 40, 3283-3295.

Elliot, D. B., \& Situ, P. (1998). Visual acuity versus letter contrast sensitivity in early cataract. Vision Research, 38, 2047-2052.

Gallant, J. L., Brau, J., \& Van Essen, D. C. (1993). Selectivity for polar hyperbolic, and cartesian gratings in macaque visual cortex. Science, 259, 100-103.

Hickey, T. L. (1977). Postnatal development of the human lateral geniculate nucleus: relationship to a critical period for the visual system. Science, $198,836-838$. 
Kaplan, H. J., Sadock, B. J., \& Sadock, V. A. (2007). Compêndio de psiquiatria: ciência do comportamento e psiquiatria clínica. Porto Alegre, RS: Artes Médicas.

Kéri, S., Antal, A., Szekeres, G., Benedek, G., \& Janka, Z. (2000). Visual information processing in patients with schizophrenia: evidence for the impairment of central mechanisms. Neuroscience Letters, 293, 69-71.

Ketomäki, J., Eloholma, M., Orreveteläinen, P., \& Halonen, L. (2003). Mesopic lighting conditions and pedestrian visibility. Ingineria Iluminatului, 11, 29-35.

Kiorpes, L., \& Movshon, J. A., (1998). Peripheral and central factors limiting the development of contrast sensitivity in macaque monkeys. Vision Research, $38,61-70$.

Licinio, J., \& Wong, M. (2007). Biologia da depressão. Porto Alegre: Artmed.

Louzã-Neto, M. R., Motta, T., Wang, Y. P., \& Elkis, H. (1995). Psiquiatria básica. Porto Alegre: Artmed.

Moreno, D. H., \& Soares, M. B. de M. (2003). Diagnósticos e tratamento: elementos de apoio - depressão. São Paulo: Lemos.

O 'Donnell, V. F., Potts, G. F., Nestor, P. G. Stylianopoulos, K. C., Shenton, M. E., \& McCarley, R. W. (2002). Spatial frequency discrimination in schizophrenia. Journal of Abnormal Psychology, 111, 620-625.

Owsley, C., Jackson, G. R., Cideciyan, A. V., Huang, Y., Fine, S. L., ... Maguire, M. G., Lolley, V., \& Jacobson, S. G. (2000). Psychophysical evidence for rod vulnerability in age-related macular degeneration. Investigative Ophthalmology and Visual Science, 41, 267-273.

Polat, U., Sagi, D., \& Norcia, A. M. (1997). Abnormal long-range spatial interactions in amblyoplia. Vision Research, 37, 737-744.

Rodrigues, M. J. S. F. (2000). O diagnóstico da depressão. Psicologia USP, 11, $1-12$.

Santos, N. A., \& Alencar, C. C. G. (2010). Early malnutrition diffusely affects children contrast sensitivity to sine-wave gratings of different spatial frequencies. Nutritional Neuroscience, 3, 1-6. doi: 10.1179/147683010x1 22611460764480
Silva, M. F., Faria, P., Regateiro, F. S., Forjaz, V., Januário, C., Freire, A., \& Castelo-Branco, M. (2005). Independent patterns of damage within magno-, parvo- and koniocellular pathways in Parkinson's disease. Brain, 128, 2260-2271.

Slaguis, W. L. (1998). Contrast sensitive for stationary and drifting spatial frequency gratings in poditie and negative symptom schizophrenia. Journal of Abnormal Psychology, 107, 49-62.

Solomon, A. (2002). O demônio do meio-dia: uma anatomia da depressão. Rio de Janeiro: Objetiva.

Szabó, Z., Antal, A., Tokaji, Z., Kálmán, J., Kéri, S., Benedek, G., \& Janka, Z. (2004). Light therapy increases visual contrast sensitivity in seasonal affective disorder. Psychiatry Research, 126, 15-21.

The World Health Report [WHO] (2001). Mental health: new understanding, new hope. Genebra, Suíça. Recuperado de http://www.who.int/whr/

Van Sluyters, R. C., Atkinson, M. S., Held, R. M., Peter Hoffman, K., \& Shatz, C. J. (1990). The development of vision and visual perception. In S. W. Spillmann \& J. S. Werner (Orgs.), The Neurophysiological Foundations (pp. 349-379). Nova Iorque: Academic Press.

Vleugels, L., Van Nunen, A., Lafosse, C., Ketelaer, P., \& Vandenbussche, E. (1998). Temporal and spatial resolution in foveal vision of multiple sclerosis patients. Vision Research, 38, 2987-2997.

Wesner, M. F., \& Tan, J. (2006). Contrast sensitivity in seasonal and nonseasonal depression. Journal of Affective Disorders, 95, 19-28. doi: 10.1016/j. jad.2006.03.028

Wetherill, G. B., \& Levitt, H. (1965). Sequential estimation of points on a psychometric function. The British Journal of Mathematical and Statistical Psychology, 18, 1-10.

Wilson, H. R. (1988). Development of spatiotemporal mechanisms in infant vision. Vision Research, 28, 611-628.

Youdelis, C., \& Hendrickson, A. (1986). A qualitative and quantitative analysis of the human fovea during development. Vision Research, 26, 847-855.

Renata Maria Toscano Barreto Lyra Nogueira, doutora em Neurociências e Comportamento pela Universidade de São Paulo, é professora Adjunta I na Universidade Federal de Alagoas (UFAL). Endereço: Campus A. C. Simões, BR 104 Norte, Km 14, Tabuleiro do Martins, CEP 57072-970, Maceió/AL - Brasil. E-mail: rm_toscano@yahoo.com.br

Natanael Antonio dos Santos, doutor em Neurociências e Comportamento pela Universidade de São Paulo, é professor Associado II na Universidade Federal da Paraíba (UFPB), coordenador do Laboratório de Percepção, Neurociências e Comportamento e bolsista de Produtividade em Pesquisa 1D pelo CNPq. E-mail: natanael_labv@yahoo.com.br 